\title{
The 56xx Desktop Micro Factory - the New Equipment for Wire-Bond Technologies*
}

\author{
J. Sedlmair and S. Berger ${ }^{\dagger}$ \\ F\&K Delvotec Semiconductor GmbH, Industriezeile 49a, A-5280 Braunau/Inn, Austria
}

F\&K Delvotec Austria, located in Braunau, is a member of the F\&K Delvotec group and focuses on bonding and testing equipment for small-scale production of high-quality electronic and semiconductor parts. The second business area concerns itself with developing and adapting bonding processes for new or unusual applications in the industry, such as bonding non-standard wire materials on non-standard surfaces, such as copper ribbon to reinforce the current-carrying capability of pc-boards.

\section{Introduction}

The Desktop Micro Factory Series 56xx from F\&K Delvotec is capable of producing quality devices like a fully automatic bonder while, at the same time, retaining the flexibility of a lab bonder in converting between different jobs. It masters all wire-bond technologies, and, in addition, offers bond testing on a single machine. The basis of the entire Desktop Factory is a highly rigid, vibration-free three-axis-gantry system with programmable, automatic motion control. This universal base fits comfortably on a lab bench, can be transported easily, and requires only a standard outlet of 110 to $240 \mathrm{~V}$ AC. All functions are controlled by a single-board PC; bond programs are created on a graphical Windows-like user surface. Thanks to an integrated network connection, they are stored not only locally on a hard disk but also remotely on any network server and can be re-imported the same way.

\section{Experimental}

The mechanicals are built around an $X-Y$ bonding table which is set in motion by robust DC servo motors and covers a bonding area of standard 100 by $100 \mathrm{~mm}$ with a positioning accuracy of better than $2 \mu \mathrm{m}$. Mechanically disconnected from this, the $\mathrm{Z}$ axis is housed in the gantry top and offers a stroke of $60 \mathrm{~mm}$ with a

\footnotetext{
* Contact person: Katarzyna Piekarska, ul.Arniki 2, 204-903 Warszawa, Poland, e-mail: kasiapiekarska@home.pl

$\dagger$ corresponding author; e-mail: stefan.berger@fkdelvotec.at
}

resolution of $1 \mu \mathrm{m}$. It features a high-precision quick-change mount for the bond or test heads. The software is straightforward and intuitive to learn. The moment a wire is programmed, it is displayed on the screen as an arrow and it can be clicked and marked using the mouse. Multiple wires can similarly be marked by mouse-click, grouped and selected after the Windows model. In this way, ultrasonic parameters can for instance be adapted. The parameter windows for each wire then show the individual values for each wire which differ from the default values - a simple way to highlight changes very clearly. Copy/paste, undo, delete functions are available just like in the customary operating systems. Once created, a bond program can be stored and retrieved at any time. Therefore the optimized bond parameters are always set correctly even if the operator changes. For small-series production the bonder works exactly like a fully automatic bonder with or without pattern recognition: the part to be bonded is clamped on the bonding table, the bond head moves to the reference marks for substrate and dies (programmed earlier) and the user corrects the exact position with the joystick. Now the bonder executes all bonds without operator assistance, taking into account all position corrections for changes in die placement. The bonder software stores all bond data, particularly the bond coordinates, in a structured query language (SQL) data bank which can be read from outside using standard SQL procedures. Especially for more complex applications this is very powerful because for future extensions CAD data (to give one example) can be imported or entire bond programs can be exported, modified externally and re-imported into the bonder. This open software architecture is especially attractive for future developments in the platform system.

The 56xx wire bonder is the very first which can be equipped with four different bond heads for all existing wire bond technologies: gold-ball bonding, thin wire wedge-wedge bonding, deep-access thin-wire wedge-wedge bonding (wire access $90^{\circ}$ ), and heavy-wire wedge-wedge bonding, also capable of bonding heavy ribbon for ultrapower applications. Each bond head is mounted and dismounted with a quick-change chuck within seconds, and each bond head contains an individual targeting miniature CCD camera so the camera magnification 
can be matched to the required resolution, and the offset between optical axis and bond tool does not have to be determined afresh after changing the bond head. All four bond heads are closely related to those working in F\&K Delvotec's fully automatic bonders. A part bonded on the 56xx can be bonded without trouble and with very little switchover time can be moved to a fully automatic bonder in larger volumes.

All bond heads supply a range of valuable quality data such as a deformation limit control (DLC) which continuously monitors the wire deformation in real time, evaluates it and displays it graphically on the screen. This allows a simple check if the wire deformation within a production lot varies more or less strongly. The 56xx family is the only worldwide machine that can be converted from a bonder to a tester and vice versa: the bond head is replaced by a test head (pull or shear tester) and the software is switched. All of this takes less than a minute. The pull and shear tester also operates in several modes, again manually and automatically. For the tester it is rather more important than for the bonder to store all measurements not only locally but also on a network so they can be monitored and evaluated by other users immediately.

A number of standard analysis tools with control cards, process capability analysis etc. are pre-installed on the tester and can be used immediately. Because a part can remain on the parts holder while the bond head is swapped against a test head, bonds can be tested immediately after being made. Especially for tricky development jobs this can be a major additional profit. The software even allows the tester to import the bond program, so programming the machine is done only once for two jobs to be performed.

That is not all for the bond tester: on top of determining pull strength and the associated force/distance diagrams it can, for the first time ever in a production machine, measure the loop height at the point of wire break. Knowing the loop height and knowing the loop length allows determining the wire geometry and therefore allows to know the angle which the wire loop forms against the horizontal plane at the time of wire break. This is extremely important because pull test values are defined for a standard wire angle of $30^{\circ}$ and they need to be re-calibrated to this angle in order to obtain values comparable to each other. In particular, for low loops (which are typical in many demanding applications), small angles of 10 to $15^{\circ}$ are customary and cause low measured pull values which cause the bonds to appear bad when in fact they are good. The only additional parameter needed for this correction is the bond length, and this is known from the bond program already in the machine. Apart from test heads for pull- and shear-tests there are further heads under development to allow peel tests which employ a plier-type gripper. They are ex- changeable in the same simple manner and are controlled by the same software.

\section{Results}

Three different pull-test heads are available with measuring ranges from $100 \mathrm{cN}$ up to $30 \mathrm{~N}$ for thin and heavy wire. Universal test head is shown in Fig. 1. All test-heads contain their calibration curves which are read out by the machine basis every time the test-head is mounted on the machine. Graphical user interface has been presented in Fig. 2.

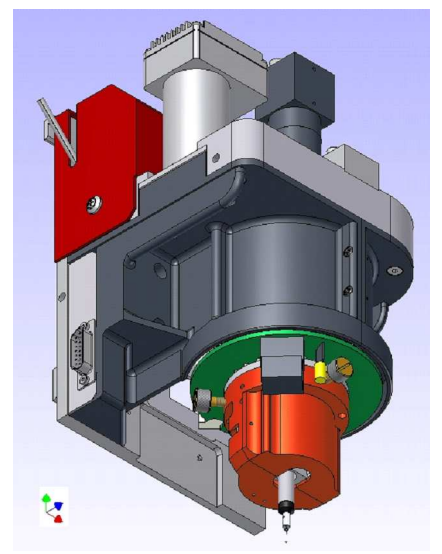

Fig. 1. Universal test head with pull-test cartridge.

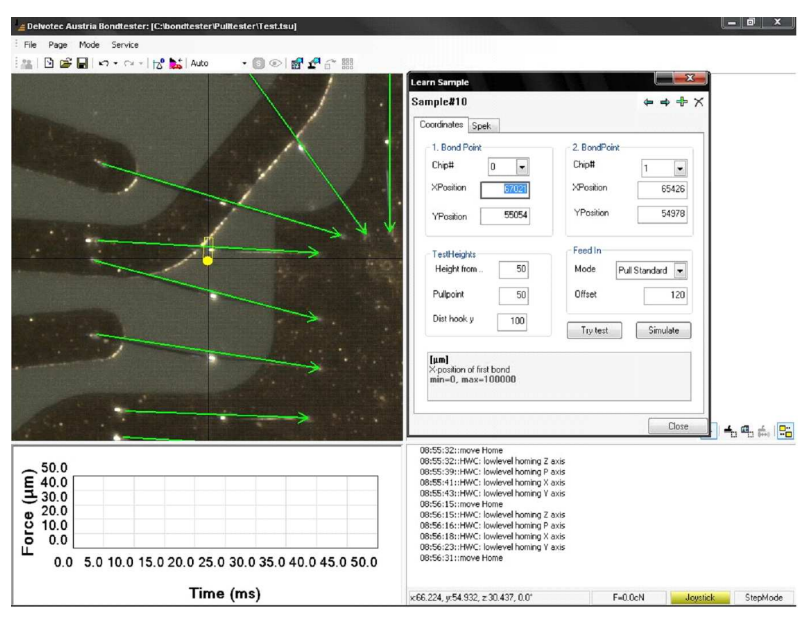

Fig. 2. Graphical user interface.

The three shear-test heads offered by F\&K Delvotec cover a measuring range of $500 \mathrm{cN}$ to $50 \mathrm{~N}$ and so they can be used not only to test wire bonds but also chip solder or adhesive connections and other mounted components such as surface mounted device parts. 